\title{
OCCUPATIONAL GENOTOXIC EFFECTS AMONG A GROUP OF NURSES EXPOSED TO ANESTHETIC GASES IN OPERATING ROOMS AT ZAGAZIG UNIVERSITY HOSPITALS
}

\author{
By \\ Borayek GE ${ }^{1}$, Abou El-Magd SA ${ }^{1}$, El-Gohary SS ${ }^{1}$, El-Naggar AM ${ }^{2}$ \\ and Hammouda $\mathrm{MA}^{1}$ \\ ${ }^{1}$ Department of Industrial Medicine and Occupational Health and ${ }^{2}$ Department of Clinical Pathology, \\ Faculty of Medicine, Zagazig University.
}

\begin{abstract}
Introduction: Waste anesthetic gases are small amounts of volatile gases that leak from the patient's anesthetic breathing circuit into the air of operating rooms during delivery of anesthesia. Personnel who are working in operating rooms are most likely to be exposed to waste anesthetic gases with no or bad automatic ventilation or scavenging systems. Aim of the work: The current study aimed to assess the level of biological marker of anesthetic gases and identify genotoxic effects among operating room nurses in Zagazig University Hospitals. Materials and methods: This study was conducted in Zagazig University Hospitals on 64 nurses who were divided into two groups: operating room nurses as exposed group, matched with outpatient clinic nurses as non exposed group. The data were collected using a questionnaire including demographic data, occupational, medical, family histories and complaints. Laboratory investigations done including Karyotyping of the chromosomes to assess genotoxic effects and assessment of urinary isoflurane. Results: Our study showed that there were highly statistical significant difference between both groups regarding occurrence of threatened and spontaneous abortion, repeated abortion $>2$ times, congenital anomalies and symptoms during work as headache and dizziness. Also this work showed that chromosomal aberrations were present among about 59\% of the exposed group, compared to $12.5 \%$ of non-exposed with highly statistically significant difference. The most common form of abnormalities were breaks of chromosomes (31.3\%), fragments $(18.8 \%)$ and polyploidy $(6.2 \%)$. There were statistical significant difference between exposed and non-exposed in number of abnormal chromosomes and urinary isoflurane.
\end{abstract}


Conclusion: Exposure to some anesthetic gases is associated with genotoxic effects among operating room personnel, leading to increased morbidity.

Key Words: Inhalational anesthetics, Halogenated anesthetic, Volatile anesthetics and Gene structure.

\section{Introduction}

Waste anesthetic gases are small amounts of volatile anesthetic gases that leak from the patient's anesthetic breathing circuit into the air of operating rooms during delivery of anesthesia. Personnel who are working in operating rooms are more likely to be exposed to waste anesthetic gases with no automatic ventilation or scavenging systems, and the latter if present are in poor condition. Also recovery rooms where gases exhaled by recovering patients are not properly vented or scavenged (Al-Ashour et al., 2014).

Volatile anesthetics are the major pollutants in operating rooms, where personnel are exposed to low doses of them, for long periods of time. Nitrous oxide and some of the halogenated anesthetics may pose a hazard to hospital workers like neurotoxic, hepatotoxic, nephrotoxic and carcinogenic effects, as well as fertility alterations, increased incidence of spontaneous abortions and congenital abnormalities have been observed. Therefore, NIOSH is concerned about workers exposed to these gases and recommends measure of control to minimize the noxious effects of anesthetic gases (NIOSH, 2007)

Experimental and epidemiological studies suggest that genotoxic effects can arise from inhalation of anesthetic gases in operating rooms, there is a great concern that operating room personnel as well as patients might be at health risks from anesthetic gases (El-ebiary et al., 2013).

Halothane (halogenated anesthetics) is used as a potent nonflammable volatile anesthetic agent. It is a haloalkane where as the structural isomers, enflurane and isoflurane, are methylethyl esters. Halogenated anesthetics are rapidly absorbed upon inhalation with a blood gas partition coefficient about 2.4 (Serkan and Calbayram, 2016).

A variable amount is metabolized in the liver by debromination and dechlorination. Up to $20 \%$ of a dose may 
be excreted in the urine as trifluroacetic acid and its salts. The bromide ion is slowly excreted in the urine. Halothane, isoflurane and enflurane are described to be modern inhalation anesthetics. However, considerable disadvantages were shown, especially the production of dose-related depression of cardiovascular and respiratory systems (Smith, 2010).

In 1999, threshold limits for environmental levels were published in the Control of Substances Hazardous to Health Regulations (COSHH) as doubts about potential adverse health effects persisted. Emphasis was placed on the need for control measures including good anaesthetic practice, good ventilation and scavenging. Despite these precautions, indoor air pollution still occur in some clinical situations, for example inhalation induction, mask ventilation and leaks around un-cuffed pediatric tracheal tubes (NIOSH, 2007).

Anesthetic gases cannot be detected by their odor until concentrations are very high. For example, halothane cannot be detected by $50 \%$ of the general population until the concentration is more than 125 times the recommended NIOSH exposure limit. (NIOSH, 2007).

\section{Aim of work}

The current study aimed to assess the level of biological marker of anesthetic gases and identify genotoxic effects among operating room nurses in Zagazig University Hospitals.

\section{Materials and methods}

- Study design: It is a comparative cross sectional study

- Place and duration of the study: The study was conducted at Zagazig University Hospitals from October 2014 to December 2016, Sharkia Governorate, Egypt.

\section{- Study Sample:}

- Sample frame: The sample frame was all nurses working in operating rooms and outpatient clinics of hospitals of Zagazig University which equal to 610 nurses.

- Sample size: The estimated sample size was calculated to be:

The exposed group: 32 operating room nurses.

The non-exposed group: 32 outpatient clinics nurse. 
- Sample selection: The sample was selected in 2 stages:

First from all hospitals (5 hospitals) exposed to general anesthesia among Zagazig University Hospitals we selected 2 hospitals by simple random method, the sample selected was:

- $\quad$ Surgery Hospital with total 200 exposed nurses.

- Cardio-thoracic Hospital with total100 exposed nurses (with total 13 operating rooms), and for the control group, we selected 2 hospitals among all non-exposed.

Second we have a list for all nurses' who are working in these hospitals of both groups, so we selected nurses by simple random method for each group considering proportional allocation technique (selecting 21 nurses from Surgery Hospital and 11 from Cardiothoracic Hospital).

\section{Subjects criteria:}

The study population included two groups:

\section{1-Exposed group}

Operating room nurses from the selected branches of Zagazig University Hospitals. Inclusion criteria:

The selected nurses had been working in Zagazig University Hospitals for more than 2 years.

Nurses who worked in operating rooms spent 6h/day and worked for 6days/week.

Willing to be involved in the study.

Exclusion criteria:

1. Nurses of the pilot were excluded from the study.

2. Nurses worked less than 2 years.

3. Nurses who had undergone medical treatment of any kind, had recent viral or bacterial infections, or worked in a service with exposure to $\mathrm{x}$-rays.

4. Nurses with known family history of genetic defects.

5. Refusing to participate in the study.

\section{2-Non-exposed group:}

It consists of nurses not exposed to anesthetic agents. They were taken from medical nurses of outpatient clinics in Zagazig University Hospitals. 


\section{Study methods:}

\section{I- A pre-designed questionnaire} was used to collect information from all participants about the following:

Part one: included questions about personal and socio-demographic data: age, sex and personal habits, as smoking.

Part two: included questions about occupational history:

- Cumulative working period (CWP).

- Position of work in operating rooms

- No. of night shifts per week and No. of working hours in each shift.

- Presence of a side job or previous job.

- Preparation of the operating room as regard anesthetic circuit and quality of scavenging or ventilation systems.

- Use of protective measures.

Part three: included questions about:

1. Any past medical problems
2. Any present medical problems related to pregnancy and labour e.g.:

- Threatened abortion.

- $\quad$ Repeated abortion and how many times occurred.

- Spontaneous abortion.

- Preterm labour.

3. Any present troubles occurred for offspring e.g.:

- Congenital anomalies.

- Still birth.

4. Any present symptoms that occurred during working day e.g.:

- Headache

- Dizziness

- Fatigue

- Syncopal attack

Part Four: included questions about:

- Family history of congenital anomalies or any genetic defects

- Consanguinity

\section{II-Laboratory investigation:}

1. Urine analysis.

2. Genotoxicity assessment. 
A sample of $2 \mathrm{ml}$ of peripheral blood was generally adequate for cytogenetic analysis. Samples were heparinized to prevent clotting, and were kept under sterile conditions until further analysis. Analysis of chromosomes aberrations was done to detect cytogenetic damage according to the method of Verma (1998).

\section{Consent}

The study group was informed about the nature and the purpose of the study and verbal consent was taken before the interview. The study group was not exposed to any harm or risk.

\section{Ethical Consideration}

The Scientific Ethical Committee of Faculty of Medicine, Zagazig University was respected (Institutional Review Board).

\section{Data management}

- The collected data were computerized and statistically analyzed using SPSS program (Statistical Package for Social Science) version 18.0.

- Qualitative data were represented as frequencies and relative percentages.

- Chi square test was used to calculate difference between qualitative variables in different groups.

- Quantitative data were expressed as Mean \pm SD (Standard deviation), median and range.

- Independent $\mathrm{T}$ test was used to calculate difference between quantitative variables in 2 groups in normally distributed data.

- Mann Whitney test was used to calculate differences between quantitative variables in 2 groups in not normally distributed data. 


\section{Results}

Table (1): Socio-demographic characteristics and occupational history of the two studied groups:

\begin{tabular}{|c|c|c|c|c|c|c|}
\hline & \multicolumn{2}{|c|}{$\begin{array}{l}\text { Group I } \\
(\text { exposed }) \\
(\text { No }=32) \\
\end{array}$} & \multicolumn{2}{|c|}{$\begin{array}{c}\text { Group II } \\
(\text { non-exposed }) \\
(\text { No }=32) \\
\end{array}$} & Test & P value \\
\hline $\begin{array}{c}\text { Age }(\text { years }) \\
\text { Mean } \pm \text { SD } \\
\text { Range }\end{array}$ & \multicolumn{2}{|c|}{$\begin{array}{c}34.9 \pm 6.5 \\
27-53 \\
\end{array}$} & \multicolumn{2}{|c|}{$\begin{array}{c}36.6 \pm 7.4 \\
25-53 \\
\end{array}$} & $0.97 \#$ & 0.34 \\
\hline $\begin{array}{ll}\text { Sex } & \\
& \text { Female } \\
\text { Male }\end{array}$ & \multicolumn{2}{|c|}{$\begin{array}{c}32(100 \%) \\
0\end{array}$} & \multicolumn{2}{|c|}{$\begin{array}{c}32(100 \%) \\
0\end{array}$} & --- & ---- \\
\hline $\begin{array}{c}\text { Working years: } \\
\text { Mean } \pm \text { SD } \\
\text { Range } \\
\end{array}$ & \multicolumn{2}{|c|}{$\begin{array}{c}17.75 \pm 5.3 \\
8-32\end{array}$} & \multicolumn{2}{|c|}{$\begin{array}{c}16.91 \pm 6.7 \\
8-31\end{array}$} & $0.56^{\wedge}$ & 0.58 \\
\hline $\begin{array}{l}\text { Cumulative working period: (hours) } \\
\text { Mean } \pm \mathrm{SD} \\
\text { Median } \\
\text { Range } \\
\end{array}$ & \multicolumn{2}{|c|}{$\begin{array}{c}46703 \pm 17735 \\
46928 \\
14080-101000\end{array}$} & \multicolumn{2}{|c|}{$\begin{array}{c}24799 \pm 11557 \\
22056 \\
6720-54000 \\
\end{array}$} & $\stackrel{\wedge}{5.002}$ & $<0.001^{* *}$ \\
\hline & No & $\%$ & No & $\%$ & $\chi^{2}$ & $\mathbf{P}$ \\
\hline $\begin{array}{l}\text { Job description : } \\
\text { Chief } \\
\text { Specified nurse } \\
\text { Nurse } \\
\text { Assistant nurse } \\
\end{array}$ & $\begin{array}{c}3 \\
10 \\
16 \\
3 \\
\end{array}$ & $\begin{array}{c}9.4 \\
31.2 \\
50 \\
9.4 \\
\end{array}$ & $\begin{array}{c}2 \\
0 \\
23 \\
7 \\
\end{array}$ & $\begin{array}{r}6.2 \\
0.0 \\
71.9 \\
21.9 \\
\end{array}$ & $13.06 \$$ & $<0.001^{* *}$ \\
\hline \multirow[t]{2}{*}{$\begin{array}{l}\text { Night shifts/week: } \\
\text { NO } \\
<4 \\
\geq 4 \\
\end{array}$} & $\begin{array}{c}3 \\
6 \\
23 \\
\end{array}$ & $\begin{array}{c}9.4 \\
18.7 \\
71.9 \\
\end{array}$ & $\begin{array}{c}16 \\
13 \\
3 \\
\end{array}$ & $\begin{array}{c}50 \\
40.6 \\
9.4 \\
\end{array}$ & $\begin{array}{c}\$ \\
25.91\end{array}$ & $<0.001^{* *}$ \\
\hline & \multicolumn{2}{|c|}{$(\mathrm{No}=\mathbf{2 9})$} & \multicolumn{2}{|c|}{$(\mathrm{No}=16)$} & & \\
\hline $\begin{array}{c}\text { Night shift hours: } \\
12 \mathrm{~h} \\
24 \mathrm{~h}\end{array}$ & $\begin{array}{c}21 \\
8\end{array}$ & $\begin{array}{l}72.4 \\
27.6\end{array}$ & $\begin{array}{c}12 \\
4\end{array}$ & $\begin{array}{l}75 \\
25\end{array}$ & $\begin{array}{c}\$ \\
1.0\end{array}$ & 0.57 \\
\hline
\end{tabular}

\#: Independent $t$ test

^: Mann Whitney test
$\$$ : Chi square test $(\chi 2)$

**: Highly significant

Table (1) shows that both Group I and Group II were matched in sociodemographic characters and there was no statistical significant difference between the two groups as regards age and sex (100\% of our sample of both groups were female), also showed that there were no statistical significant difference between both groups as regards No. of working years but there were highly statistical significant difference between them in cumulative working period (CWP) and number of night shifts worked /week with marked increase among Group I 9 the exposed group). 
Table (2): Medical history and health status of the two studied groups:

\begin{tabular}{|c|c|c|c|c|c|c|}
\hline \multirow[b]{2}{*}{ Variables } & \multicolumn{2}{|c|}{$\begin{array}{c}\text { Group I } \\
(\text { No }=32)\end{array}$} & \multicolumn{2}{|c|}{$\begin{array}{l}\text { Group II } \\
(\text { No = 32) }\end{array}$} & \multirow[t]{2}{*}{$\mathbf{P}^{\$}$} & \multirow[t]{2}{*}{ OR } \\
\hline & No & $\%$ & No & $\%$ & & \\
\hline $\begin{array}{l}\text { Previous diseases: } \\
\text { NO } \\
\text { Yes }\end{array}$ & $\begin{array}{c}32 \\
0\end{array}$ & $\begin{array}{c}100 \\
0\end{array}$ & $\begin{array}{c}30 \\
2 \\
\end{array}$ & $\begin{array}{c}93.8 \\
6.2 \\
\end{array}$ & 0.15 & ------ \\
\hline $\begin{array}{l}\text { Threatened abortion: } \\
\text { NO } \\
\text { Yes }\end{array}$ & $\begin{array}{l}21 \\
11\end{array}$ & $\begin{array}{l}65.6 \\
34.4\end{array}$ & $\begin{array}{c}28 \\
4\end{array}$ & $\begin{array}{l}87.5 \\
12.5\end{array}$ & $<0.05^{*}$ & $\begin{array}{c}2.89 \\
(1.07-8.84)\end{array}$ \\
\hline $\begin{array}{l}\text { Spontaneous abortion: } \\
\text { NO } \\
\text { Yes }\end{array}$ & $\begin{array}{l}17 \\
15\end{array}$ & $\begin{array}{l}53.1 \\
46.9\end{array}$ & $\begin{array}{c}31 \\
1\end{array}$ & $\begin{array}{c}96.9 \\
3.1\end{array}$ & $<0.001^{* *}$ & $4.57(1.83-33.2)$ \\
\hline $\begin{array}{l}\text { Repeated abortion>2 times: } \\
\text { NO } \\
\text { Yes } \\
\end{array}$ & $\begin{array}{c}26 \\
6 \\
\end{array}$ & $\begin{array}{l}81.2 \\
18.8 \\
\end{array}$ & $\begin{array}{c}32 \\
0 \\
\end{array}$ & $\begin{array}{l}100 \\
0.0 \\
\end{array}$ & $<0.05^{*}$ & $\begin{array}{c}2.76 \\
(1.1-6.51) \\
\end{array}$ \\
\hline $\begin{array}{c}\text { Congenital anomalies of } \\
\text { offspring: } \\
\text { NO } \\
\text { Yes }\end{array}$ & $\begin{array}{c}23 \\
9\end{array}$ & $\begin{array}{l}71.9 \\
28.1\end{array}$ & $\begin{array}{c}31 \\
1\end{array}$ & $\begin{array}{c}96.9 \\
3.1\end{array}$ & $<0.001^{* *}$ & $2.62(1.04-7.23)$ \\
\hline $\begin{array}{l}\text { Types of anomalies: } \\
\text { Congenital heart }\end{array}$ & $\begin{array}{l}5 \\
3\end{array}$ & $\begin{array}{l}55.6 \\
33.3\end{array}$ & $\begin{array}{l}1 \\
0\end{array}$ & $\begin{array}{l}100 \\
0.0\end{array}$ & $\begin{array}{l}0.39 \\
0.49\end{array}$ & $\begin{array}{c}0.844(0.58-1.19) \\
1.17(0.86-1.58)\end{array}$ \\
\hline $\begin{array}{c}\text { Congenital Hydrocephalus } \\
\text { Congenital brain tumor }\end{array}$ & 1 & 11.1 & 0 & 0.0 & 0.73 & $1.13(0.89-1.42)$ \\
\hline $\begin{array}{l}\text { Suffering from symptoms } \\
\text { during working: } \\
\text { NO } \\
\text { Yes }\end{array}$ & $\begin{array}{c}7 \\
25\end{array}$ & $\begin{array}{l}21.2 \\
78.1\end{array}$ & $\begin{array}{c}29 \\
3\end{array}$ & $\begin{array}{c}90.6 \\
9.4\end{array}$ & $\begin{array}{l}<0.001^{* *} \\
<0.001^{* *}\end{array}$ & $4.88(1.83-30.1)$ \\
\hline $\begin{array}{l}\text { Headache } \\
\text { Dizziness } \\
\text { Fatigue } \\
\text { Syncopal attack }\end{array}$ & $\begin{array}{c}23 \\
22 \\
21 \\
5\end{array}$ & $\begin{array}{l}92 \\
88 \\
84 \\
20\end{array}$ & $\begin{array}{l}1 \\
1 \\
2 \\
0\end{array}$ & $\begin{array}{c}33.3 \\
33.3 \\
66.4 \\
0.0\end{array}$ & $\begin{array}{c}<\mathbf{0 . 0 5} * \\
0.45 \\
0.59\end{array}$ & $\begin{array}{l}4.24(1.28-14.8) \\
2.85(1.09-9.36) \\
1.66(0.55-5.04) \\
2.09(0.31-7.36)\end{array}$ \\
\hline
\end{tabular}

** Highly statistically significant

*Significant

$\$$ test of significance is fisher exact

Table (2) showed that there was no statistical significant difference between Group I and Group II as regards previous diseases (as recent viral or bacterial infections). But there was highly statistical significance difference between them in occurrence of threatened and spontaneous abortion, repeated abortion $>2$ times, congenital anomalies and symptoms during work (headache, dizziness, fatigue and syncopal attacks) with marked increase among Group I. 
Table (3): Types of chromosomal abnormalities detected among the two studied groups:

\begin{tabular}{|c|c|c|c|c|c|c|c|}
\hline \multirow[t]{2}{*}{ Variables } & \multicolumn{2}{|c|}{$\begin{array}{c}\text { Group I } \\
(\text { No }=32)\end{array}$} & \multicolumn{2}{|c|}{$\begin{array}{l}\text { Group II } \\
(\text { No = 32) }\end{array}$} & \multirow[t]{2}{*}{ Test } & \multirow[t]{2}{*}{$p$ value } & \multirow[t]{2}{*}{ OR } \\
\hline & No & $\%$ & No & $\%$ & & & \\
\hline $\begin{array}{l}\text { Chromosomal abnormalities: } \\
\text { NO } \\
\text { Yes }\end{array}$ & $\begin{array}{l}13 \\
19\end{array}$ & $\begin{array}{l}40.6 \\
59.4\end{array}$ & $\begin{array}{c}28 \\
4\end{array}$ & $\begin{array}{l}87.5 \\
12.5\end{array}$ & $15.27^{\#}$ & $<0.001^{* *}$ & $\begin{array}{c}4.52 \\
(1.8-11.49)\end{array}$ \\
\hline $\begin{array}{l}\text { Type of abnormalities: } \\
\text { Breaks } \\
\text { Fragments } \\
\text { Polyploidy }\end{array}$ & $\begin{array}{l}10 \\
6 \\
3\end{array}$ & $\begin{array}{l}31.3 \\
18.8 \\
6.2\end{array}$ & $\begin{array}{l}4 \\
0 \\
0\end{array}$ & $\begin{array}{l}12.5 \\
0.0 \\
0.0\end{array}$ & $\begin{array}{c}\text { Fisher } \\
\text { exact }\end{array}$ & $\begin{array}{l}<0.05^{*} \\
<0.05^{*} \\
0.301\end{array}$ & $\begin{array}{c}2.47(1.86-7.23) \\
2.55(1.93-9.88) \\
1.43(1.22-3.65)\end{array}$ \\
\hline $\begin{array}{l}\text { Number of chromosomes: } \\
\text { Mean } \pm \text { SD } \\
\text { Median } \\
\text { Range }\end{array}$ & \multicolumn{2}{|c|}{$\begin{array}{c}3.2 \pm 1.2 \\
3 \\
1-5\end{array}$} & \multicolumn{2}{|c|}{$\begin{array}{c}2 \pm 0.82 \\
2 \\
1-3\end{array}$} & $2.9^{\wedge}$ & $<0.05^{*}$ & \\
\hline $\begin{array}{l}\text { Urinary isoflurane }(\mu \mathrm{g} / \mathrm{L}) \\
\text { Mean } \pm \text { SD } \\
\text { Median } \\
\text { Range }\end{array}$ & \multicolumn{2}{|c|}{$\begin{array}{c}3.4 \pm 1.07 \\
3.3 \\
2-6.4\end{array}$} & \multicolumn{2}{|c|}{$\begin{array}{l}0.28 \pm 0.24 \\
0.2 \\
0.11-1.1\end{array}$} & $6.8^{\wedge}$ & $<0.001^{* *}$ & \\
\hline
\end{tabular}

** Highly statistically significant

*Significant

\# Chi square test $(\chi 2)$

^: Mann Whitney test

Table (3) showed that chromosomal aberrations were present among about 59\% of Group I (exposed), compared to $12.5 \%$ of Group II (non-exposed) with highly statistically significant difference. The most common form of abnormalities were breaks of chromosomes followed by fragments and polyploidy which was present only among Group I. There was statistical significant difference between both groups as regards number of abnormal chromosomes and urinary isoflurane with marked elevation of the latter among Group I. 
Table (4): Correlation between chromosomal aberrations and urinary isoflurane with age and items of occupational history among Group I:

\begin{tabular}{|l|c|c|c|c|}
\hline \multirow{2}{*}{} & \multicolumn{2}{|c|}{$\begin{array}{c}\text { Chromosomal } \\
\text { aberrations }\end{array}$} & \multicolumn{2}{c|}{ Urinary isoflurane } \\
\cline { 2 - 5 } & $\mathbf{r}$ & $\mathbf{p}$ value & $\mathbf{r}$ & $\mathbf{p}$ value \\
\hline \multirow{2}{*}{ Age/ years } & 0.322 & 0.179 & 0.296 & 0.101 \\
\hline Working years & 0.891 & $<\mathbf{0 . 0 0 1} * *$ & 0.250 & 0.167 \\
\hline Cumulative working period/ years & 0.698 & $<\mathbf{0 . 0 0 1} * *$ & 0.474 & $<\mathbf{0 . 0 0 1} * *$ \\
\hline No of shifts/week & 0.734 & $<\mathbf{0 . 0 0 1} * *$ & 0.412 & $<\mathbf{0 . 0 0 1 * *}$ \\
\hline Hours of shift & 0.665 & $<\mathbf{0 . 0 0 1} * *$ & 0.406 & $<\mathbf{0 . 0 5 *}$ \\
\hline
\end{tabular}

** Highly statistically significant

*Significant

Table (4) showed a positive correlation between chromosomal aberrations, urinary isoflurane and age but it didn't reach a significant level. Chromosomal aberrations showed a highly statistically significant positive correlation with working/ years, cumulative working period, No of shifts/week and hours of shift. Urinary isoflurane showed a statistically significant positive correlation with cumulative working period, No of shifts/week and hours of shift. 
Table (5): Relation between chromosomal aberrations, occupational history and medical problems among Group I:

\begin{tabular}{|c|c|c|c|c|c|c|}
\hline Variables & \multicolumn{2}{|c|}{$\begin{array}{c}\text { Present } \\
(\text { No }=19)\end{array}$} & \multicolumn{2}{|c|}{$\begin{array}{c}\text { Absent } \\
(\mathbf{N o}=13)\end{array}$} & Test & $p$ value \\
\hline $\begin{array}{l}\text { Working years: } \\
\text { Mean } \pm \text { SD } \\
\text { Range } \\
\end{array}$ & \multicolumn{2}{|c|}{$\begin{array}{c}21.1 \pm 5.84 \\
12-32\end{array}$} & \multicolumn{2}{|c|}{$\begin{array}{c}13.38 \pm 2.84 \\
8-18\end{array}$} & $4.37^{\#}$ & $<0.001$ ** \\
\hline $\begin{array}{l}\text { Cumulative working period/hours } \\
\text { Mean } \pm \text { SD } \\
\text { Median } \\
\text { Range } \\
\end{array}$ & \multicolumn{2}{|c|}{$\begin{array}{c}49867 \pm 19457.5 \\
459360 \\
25800-101000\end{array}$} & \multicolumn{2}{|c|}{\begin{tabular}{|c}
$25171 \pm 9934.3$ \\
21840 \\
$14080-47040$
\end{tabular}} & $3.86^{\wedge}$ & $<0.001^{* *}$ \\
\hline & No & $\%$ & No & $\%$ & test & $\bar{p}$ \\
\hline $\begin{array}{l}\text { Night shifts/week: } \\
\text { NO } \\
<4 \\
\geq 4\end{array}$ & $\begin{array}{c}2 \\
0 \\
17\end{array}$ & $\begin{array}{l}10.5 \\
0.0 \\
89.5\end{array}$ & $\begin{array}{l}1 \\
6 \\
6\end{array}$ & $\begin{array}{l}7.6 \\
46.2 \\
46.2\end{array}$ & $10.85^{\$}$ & $<0.001^{* *}$ \\
\hline $\begin{array}{l}\text { Using PPE: } \\
\text { Yes } \\
\text { No }\end{array}$ & $\begin{array}{c}12 \\
7 \\
\end{array}$ & $\begin{array}{l}63.2 \\
36.8 \\
\end{array}$ & $\begin{array}{c}10 \\
3\end{array}$ & $\begin{array}{l}76.9 \\
23.1 \\
\end{array}$ & $* * *$ & 0.34 \\
\hline & \multicolumn{2}{|c|}{$\begin{array}{c}150.8 \\
(\mathbf{N o}=\mathbf{1 7}) \\
\end{array}$} & \multicolumn{2}{|c|}{$\begin{array}{l}3 \\
\text { No }=12) .1\end{array}$} & & \\
\hline $\begin{array}{l}\text { Shift hours: } \\
12 \mathrm{~h} \\
24 \mathrm{~h} \\
\end{array}$ & $\begin{array}{l}9 \\
8 \\
\end{array}$ & $\begin{array}{l}52.9 \\
47.1 \\
\end{array}$ & $\begin{array}{c}12 \\
0 \\
\end{array}$ & $\begin{array}{l}100 \\
0.0 \\
\end{array}$ & $* * *$ & $<0.05^{*}$ \\
\hline $\begin{array}{l}\text { Spontaneous abortion: } \\
\text { NO } \\
\text { Yes } \\
\end{array}$ & $\begin{array}{c}6 \\
13 \\
\end{array}$ & $\begin{array}{l}31.6 \\
68.4 \\
\end{array}$ & $\begin{array}{c}11 \\
2\end{array}$ & $\begin{array}{l}84.6 \\
15.4 \\
\end{array}$ & $* * *$ & $<0.001 * *$ \\
\hline $\begin{array}{l}\text { Repeated abortion >2 times: } \\
\text { NO } \\
\text { Yes } \\
\end{array}$ & $\begin{array}{c}13 \\
6 \\
\end{array}$ & $\begin{array}{l}68.4 \\
31.6 \\
\end{array}$ & $\begin{array}{c}13 \\
0 \\
\end{array}$ & $\begin{array}{l}100 \\
0.0\end{array}$ & $* * *$ & $<0.05^{*}$ \\
\hline $\begin{array}{l}\text { Congenital anomalies of offspring: } \\
\text { NO } \\
\text { Yes }\end{array}$ & $\begin{array}{c}11 \\
8\end{array}$ & $\begin{array}{l}57.9 \\
42.1\end{array}$ & $\begin{array}{c}12 \\
1\end{array}$ & $\begin{array}{c}92.3 \\
7.7\end{array}$ & *** & $<0.05^{*}$ \\
\hline $\begin{array}{l}\text { Types : } \\
\text { Congenital heart diseases } \\
\text { Congenital hydrocephalus } \\
\text { Congenital brain tumor } \\
\end{array}$ & $\begin{array}{l}4 \\
3 \\
1 \\
\end{array}$ & $\begin{array}{c}50 \\
37.5 \\
12.5 \\
\end{array}$ & $\begin{array}{l}1 \\
0 \\
0 \\
\end{array}$ & $\begin{array}{l}100 \\
0.0 \\
0.0\end{array}$ & $* * *$ & $\begin{array}{l}0.343 \\
0.453 \\
0.708 \\
\end{array}$ \\
\hline $\begin{array}{l}\text { Suffering from symptoms during work: } \\
\text { NO } \\
\text { Yes }\end{array}$ & $\begin{array}{c}4 \\
15\end{array}$ & $\begin{array}{l}21.1 \\
78.9 \\
\end{array}$ & $\begin{array}{c}3 \\
10 \\
\end{array}$ & $\begin{array}{l}23.1 \\
76.9\end{array}$ & **** & 0.28 \\
\hline
\end{tabular}

\#: Independent t test $\quad \$$ : Chi square test $(\chi 2) \quad \wedge$ : Mann Whitney test $\quad * * *$ : fisher exact test **: Highly significant $\quad *$ : statistical significant

Table (5) showed a statistical significant difference between nurses with chromosomal aberrations and nurses without chromosomal aberrations as regards working years, cumulative working period (CWP), No of night shifts and night shift hours with increase all among nurses with chromosomal aberrations. Also, there was statistical significant difference between both groups as regards occurrence of repeated abortion $>2$ times, spontaneous abortion and congenital anomalies. 


\section{Discussion}

There is a great concern that the operating room personnel might be exposed to health risks due to exposure to anesthetic gases. However, whether chronic exposure to these gases is hazardous to the health of anesthetic room personnel is still controversial (Dittmar et al., 2015).

This study was conducted in Zagazig University Hospitals on 64 nurses who were divided into two groups: operating room nurses (32) and outpatient clinic nurses (32).

Our study showed that both groups were matched in sociodemographic data with mean age 35 years in Group I and 37 years in Group II and with no statistical significant difference. Regarding sex both groups were $100 \%$ females, this is may be because females represent more than $95 \%$ of nursing sector of Zagazig University Hospitals (Table 1).

Our study found that there was no statistical significant difference between Group I and Group II as regards the presence of previous diseases (as viral or bacterial infections). But there was highly statistical significant difference between them in occurrence of threatened and spontaneous abortion, abortion $>2$ times, congenital anomalies and symptoms during work as headache and drowsiness with marked increase among Group I (Table 2). The present study showed that $92 \%$ of exposed nurses were suffering from headache during work in contrast to $33.3 \%$ among the non exposed group, and $88 \%$ of operating room nurses (ORP) were complaining of dizziness (Table 2).This is consistent with a study carried out by Al-Ashour et al. (2014) who detected that $(55.2 \%)$ of his studied group were suffering from headache and (69\%) were suffering from dizziness.

In another study carried out on operating room personnel, Abd El-Aal et al. (2008) revealed higher prevalence of dizziness, headache, irritability, decreased concentration, anxiety and easy fatigability compared to controls.

Occupational Safety and Environmental Health (OSEH) (2007) reported that workers exposed to excess amounts of anesthetic gas can experience symptoms of drowsiness, headache, nausea, poor judgment and loss of coordination. 
Our results revealed that about $28 \%$ of exposed group had congenital anomalies of their offspring compared to $3.1 \%$ among their controls with OR 2.6 95\% CI (1.04-7.23) higher among exposed (Table 2). This is similar with a retrospective cohort study carried out by Teschke et al. (2011) which proved that anomalies were associated maternal exposure to halogenated gases (ORs: 1.49, 95\% CI: 1.04-2.13; and 2.61, 95\% CI: 1.31-5.18, respectively). Anomalies most frequently associated with exposure were those of the heart (OR, halogenated gases: 2.31, 95\% CI: 1.07-4.97) and skin integumentery system (epidermis and dermis e.g.: icthyosis) (OR, halogenated gases: 3.56, 95\% CI: 1.53-8.32; OR, nitrous oxide: $3.02,95 \%$ CI: $1.37-6.64)$.

Also the current study showed that the most frequent anomaly was that of the heart and the risk among nurses exposed to halogenated anaesthetics was (OR, 0.84, 95\% CI:0.58-1.19), but the risk was higher for congenital hydrocephalus (OR, 1.17, 95\% CI: 0.86-1.58) (Table 2).

The higher risk of congenital anomalies among our group may be explained as we depend only on medical history from the nurses and not confirm the results with karyotyping for offsprings to prove the presence of congenital anomalies.

Our work was in agreement with a study carried out by Mohammed and Khalid (2016), who found that the prevalence of congenital anomalies among offspring of female workers exposed to waste anesthetic gases was $3.3 \%$, while among their controls was $0 \%$ with relative risk $(0.81,95 \% \mathrm{CI}$ : $0.75-1$ ), also they found that $46.7 \%$ of exposed females suffered from abortion.

Our study showed that chromosomal aberrations were present among about $59 \%$ of exposed group, compared to $12.5 \%$ among non-exposed with highly statistical significant difference. The most common form of abnormalities were breaks of chromosomes $(31.3 \%)$, followed by fragments (18.8\%) and polyploidy $(6.2 \%)$ which were present only among the exposed group (Group I) (Table 3).

This is consistent with the results obtained by Abd El-Aal et al. (2008) on his study on DNA fragmentation which revealed statistically significant 
increase in the mean value of optical density of DNA fragment in leukocytes of operating room personnel (ORP), compared to controls. Also the current results were similar with the study done by Rozgaj et al. (2009) who found that the group exposed to anesthetic gases revealed significant increase in genome damage in both the comet assay test and micronucleus frequency test compared to their controls.

On his study on chromosomal aberrations (CA) among nurses exposed to anesthetic gazes, Aldrieny et al. (2013) found that the control group showed four types of the (CA), however, the exposed personnel metaphases showed many types of structural aberrations that appeared singly or in combination inside the same cell. All types of chromosomal aberrations showed a statistically significant increase among the exposed group with respect to controls except for dicentric chromosomes as the difference was not significant. Chromosome gaps were the most frequent chromosomal aberrations observed in both groups with mean $(4.923 \pm 2.682$ and 0.846 \pm 0.128 ) for the exposed and control groups respectively, followed by the second frequent chromosomal aberration which was the acentric fragments. Chromosome breaks were also detected to be significantly higher among the exposed group compared to their controls $(1.269 \pm 1.282$ and 0.769 \pm 1.230 respectively).

Izdes et al. (2009) assessed the possible genotoxic risk, by the alkaline comet assay, in the peripheral blood lymphocytes of nurses who are exposed to waste anesthetic gases. He pointed to the potential risk of DNA damage in nurses who are exposed to waste anesthetic gases

In our study, there was statistical significant difference between exposed and non-exposed in number of abnormal chromosomes $(3.2 \pm 1.2$ versus $2 \pm 0.82$ respectively) and urinary isoflurane with marked elevation of urinary isoflurane among the exposed group (Table 3).

This is in agreement with Shaker et al. (2011) who revealed that there was a significant increase in total chromosomal aberrations among the operating room nurses, indicating chromosomal damage when compared with controls $(4.3 \pm 3.3$ versus $2.3 \pm 1.4 ; \mathrm{p}<0.05)$. 
This is also consistent with Aldrieny et al. (2013), who stated that in the operating room personnel, there was a significant increase in the total chromosomal aberrations, when compared with controls $(\mathrm{P}<0.05)$ with mean of $10.7 \pm 4.99$ total chromosomal aberrations compared to $4 \pm 4.39$ among their controls.

Our study showed a positive correlation between chromosomal aberrations and age but it did not reach a significant level. Also, chromosomal aberrations showed statistically significant positive correlation with working years, cumulative working period, No of shifts/week and hours of shift. Urinary isoflurane showed a statistically significant positive correlation with cumulative working period, No of shiftslweek and hours of shift (Table 4). This is consistent with Shaker et al. (2011) who detected a positive correlation between chromosomal aberrations and age but it did not reach a significant level, and also years of exposure which did not reach a significant level on contrary to our results.
Also, in agreement with our results, Abd El-Aal et al. (2008) who revealed a significant positive correlation between DNA fragmentation and duration of exposure to volatile anesthetics and Rozgaj et al. (2009) who reported significant correlation between duration of exposure and genetic damage induced by anesthetic gases in occupationally exposed populations.

In our study, there was statistical significant difference between nurses with and without chromosomal aberrations as regards working years, CWP, No of night shifts and shift hours with increase in all these parameters among nurses with abnormal chromosomes (Table 5).Also there were statistical significant difference between nurses with and without chromosomal aberrations as regard occurrence of spontaneous abortion, repeated abortion $>2$ times and congenital anomalies (Table 5).

Dittmar et al. (2015) confirmed that there was a genetic damage due to exposure to inhaled anesthetics which was significant in woman and not in men. Also, Rozgaj et al. (2009) reported that there was a significant increased 
relative risk values for chromosomal aberrations and micronucleus for woman.

Shaker et al. (2011) estimated the genotoxic risk of occupational exposure to anesthetic gases in a group of operating room nurses who were examined by conventional cytogenetic methods: Chromosomal aberrations analysis and Sister Chromatid Exchange analysis, and investigations for the possible relation of these findings with age and duration of exposure. They concluded that exposure to even low concentrations of waste anesthetic gases may result in an increased risk of genetic damage which may lead to increased morbidity.

Musak et al. (2017) quantified the association between the occupational exposure of physicians and nurses employed in operating rooms (exposure to volatile anesthetics), and the occurrence of chromosomal aberrations (CA) compared with sex- and agematched controls. They indicated that the presence of genotoxic compounds in operating rooms, results in a significant increase of chromosomal damage (impairment of chromosomal integrity) among the medical workers employed in these facilities.

de Araujo et al. (2013) verified the frequency of mono-nucleated lymphocytes among professionals who were occupationally exposed to anesthetic gases in operating rooms and compared with professionals non-exposed in other hospital areas of the same hospital. They detected an increase in micronucleus (MN) frequency in bi-nucleated $(\mathrm{BN})$ cells among the exposed group compared to the control. Therefore, the age and period of working time in an operating room influenced the micronucleus (MN) frequency only in women professionals (de Araujo et al., 2013).

\section{Conclusion}

The results obtained from this study indicated that occupational exposure to halogenated anesthetic agents induced an increase in the level of genotoxicity which was significant in the frequency of chromosomal aberrations.

Besides the genotoxic damage seen in the operating room personnel, some other effects of exposure to waste anesthetic gases were reported 
such as dizziness, headache, fatigue and irritability, as well as miscarriages among operating room nurses and congenital abnormalities in their offspring. This outcome associated with our poorly equipped operating rooms (not having a central high-flow scavenging system and low leakage anesthesia machines, and not having facilities to use low-flow and closedcircuit anesthesia).

We concluded that exposure to some anesthetic gases is associated with genotoxic effects among operating room personnel, leading to increased morbidity.

\section{Conflict of interest}

Authors have declared that no conflict of interest exists.

\section{References}

1. Abd El-Aal BG, Al-Batanony MA and ElShafiy MK (2008): Genotoxic and oxidative stress effects due to occupational exposure to anesthetic gases among operating room personnel. Menoufiya Medical Journal; 21(1).

2. Al-Ashour IA, Abd-Ali DK, Fallah MA and Kteo IQ (2014): Effect of inhaled anesthetic gases on health staff health status in ALNajaf City. International $\mathbf{J}$ of Scientific and Technology Research ;3:12.

3. Aldrieny EA, Abd-El-Hafez AAA and Abd-ElHafez AAA (2013): Effects of wasted anesthetic gases on human lymphocytes: A genetic study. Journal of Microscopy and Ultrastructure; 1: 89-95.
4. de Araujo TK, da Silva-Grecco, Bisinotto F M, Roso NC and Pissetti CW (2013): Genotoxic effects of anesthetics in operating room personnel evaluated by micronucleus test. Journal of Anesthesiology and Clinical Science; ISSN 2049-9756:2-26.

5. Dittmar M, Petermichl W, Lindner R and Sinner $B$, et al (2015): In Vitro Induction of Endothelial Apoptosis of the Post-Hypoxic Blood-Brain Barrier by isoflurane but Not by Sevoflurane and Midazolam. PLoS One; 10 (6).

6. El-ebiary AA, Abu Elfadl AA and Sarhan NI (2013): Assessment of genotoxicity risk in operation room personnel by the alkaline comet assay. Human and HYPERLINK "https://www.researchgate.net/ journal/1477-0903_Human_Experimental_ Toxicology"andandHYPERLINK "https:// Www.researchgate.net/journal/1477-0903 Human_Experimental_Toxicology" Experimental Toxicology ; 70(6): 32-563.

7. Izdes S, Sardas S, Kadioglu E, Kaymak C and Ozcagli E (2009): Assessment of genotoxic damage in nurses occupationally exposed to anaesthetic gases or antineoplastic drugs by the comet assay. J Occup Health; 51: 283-6

8. Mohammed NA and Khalid AA (2016): Occupational Hazards Associated with Exposure to Anesthetic Gases on Reproductive Health in Operating Theatre Staff in Three Hospitals in Khartoum State, Sudan Department of Anesthesia Technology. Available from: https// pdfs.semanticscholar.org/0a9b/0a23540e96d6d 482a26bc9c0b0b3b93a9bb8.pdf.

9. Musak L, Smerhovsky Z, Halasova E, Osina O, et al. (2013): Chromosomal damage among medical staff occupationally exposed to volatile anesthetics, antineoplastic drugs, and formaldehyde. Scand J Work Environ Health; 39(6): 618-30.

10. NIOSH (2007): Publication No. 151: Waste Anesthetic Gases-Occupational Hazards in Hospitals.

11. Occupational Safety and Environmental Health Guideline (OSEH) (2007): www.OSEH.com. 
12. Rozgaj R, Kasuba V, Brozovic G and Jazbec A (2009): Genotoxic effects of anaesthetics in operating theatre personnel evaluated by the comet assay and micronucleus test. Int $\mathrm{J}$ Hyg Environ Health; 212: 11-7.

13. Serkan Y and Calbayram C (2016): Exposure to anesthetic gases among operating room personnel and risk of genotoxicity: A systematic review of the human biomonitoring studies? Journal of Clinical Anesthesia; 35: 326-31.

14. Shaker DA, Samir AM, Hagag HA, Abd El-Aal AA and Afify RA (2001): Cytogenetic damage in operating room nurses exposed to anesthetic gases. Med J Cairo Univ; 79(1): 237-44.
15. Smith FD (2010): Management of exposure to waste anesthetic gases. AORN Journal; 4: 48291.

16. Teschke K, Abanto Z, Arbour L, Beking K, et al. (2011): Exposure to anesthetic gases and congenital anomalies in offspring of female registered nurses. American Journal of Industrial Medicine; 54(2): 118-27.

17. Verma A (1998): Cytogenetic investigations on patients with oral submucous fibrosis. Ind Med Assoc; 96(2): 51-7. 